BULL. AUSTRAL. MATH, SOC.

VOL. 33 (1986), 177-187.

\title{
SYMYETPY GROUPS ON ORDERED BANACH SPACES
}

\author{
SADAYUKI YAMAMURO
}

A symmetry of an ordered Banach space is an order and norm isomorphism which commutes with its ideal centre. A class of ordered Banach spaces is introduced to show that, for a space in this class, the group of symmetries is trivial if and only if the space is lattice-ordered. When this group becomes larger, the space approaches an antilattice. This phenomenon is also investigated.

\section{Preliminaries.}

Let $B$ be a real Banach space ordered by a closed and proper positive cone $B^{+}$. Throughout this paper, $B$ is always assumed to be archimedean.

The cannonical half-norm $N$ associated with $B^{+}$, due to [3], is defined by

$$
N(x)=\inf \left\{\|x+y\|: y \in B^{+}\right\} \text {for all } x \in B \text {. }
$$

An element $x \in B$ is said to be orthogonally decomposable if there exist elements $y$ and $z$ of $B^{+}$such that

Reseived 24 May 1985.

Copyright Clearance Centre, Inc. Serial-fee code: 0004-9727/86 $\$ A 2.00+0.00$. 


$$
x=y-z, \quad\|y\|=N(x) \text { and }\|z\|=N(-x) .
$$

If every element of $B$ is orthogonally decomposable, then $B$ is said to be orthogonally decomposable. (See [17].)

Let $L(B)$ be the Banach space of all continuous linear operators on $B$ with the operator bound norm. The positive cone $L(B)^{+}$consists of $\phi$ in $L(B)$ such that $\phi\left(B^{+}\right) \subset B^{+} . B$ is said to have the Robinson property if

$$
\|\phi\|=\sup \left\{\|\phi(x)\|:\|x\| \leq 1, x \in B^{+}\right\}
$$

for all $\phi \in L(B)^{+}$. (See [16].)

An $N$-automorphism of $B$ is a bijective element $\phi$ of $L(B)$ such that $N(\phi(x))=N(x)$ for all $x \in B$. The set of all N-automorphisms of $B$ is deonted by $G(B)$, which is obviously a group. The following two facts have been proved in [17].

(1.1) When $B$ is orthogonaliy decomposable, $\phi \in G(B)$ if and only if $\|\phi(x)\|=\|x\|$ for all $x \in B^{+}$and $\phi$ is an 0 . d. isomorphism (that is, $\phi$ is a continuous bijection and $\phi(x)=\phi(y)-\phi(z)$ is an orthogonal decomposition of $\phi(x)$ if and only if $x=y-z$ is an orthogonal decomposition of $x$ ).

(1.2) When $B$ is orthogonally decomposable and has the Robinson property, then $\phi \in G(B)$ if and only if $\phi$ is a bipositive isometry. The ideal centre of $B$ is the set $Z(B)$ of all elements $T$ of $L(B)$ such that there exists a number $\lambda$, depending on $T$, such that $-\lambda x \leq T x \leq \lambda x$ for all $x \in B^{+}$. For $T \in Z(B)$, we can define a norm

$$
\|T\|_{0}=\inf \left\{\lambda \geq 0:-\lambda x \leq T x \leq \lambda x \text { for all } x \in B^{+}\right\} .
$$

A sufficient condition for $\|T\|_{0}=\|T\|$ for all $T \in Z(B)$ is that both the norms of $B$ and $B^{*}$, the dual of $B$, are absolutely monotone. (See [15], Lemma 2.3 and [4], Theorem 1.3.1.) If this is the case, Z(B) is an ordered Banach space with an archimedean order and the multiplicative unit. Hence, by [10], it is an abelian real Banach algebra. For the spectrum $\Omega$ of $Z(B)$, the Gelfand transform is an isometric, order and algebraic isomorphism onto $C(\Omega)$. Furthermore, by [15], Corollary 1.13, for every $a \in B^{+}$, the map $T \leftrightarrow T a$ is a lattice homomorphism of $Z(B)$ 
onto a sublattice of $B$. Following [15], we call $B$ regular if $\|T\|_{0}=\|T\|$ for all $T \in Z(B)$.

An element $\phi$ of $G(B)$ such that $\phi T=T \phi$ for all $T \in Z(B)$ is called a symmetry. (See [7] and [8].) The set of all symmetries is denoted by $S(B)$, which is obviously a subgroup of $G(B)$.

The positive cone $\left(B^{*}\right)^{+}$of the dual $\bar{B}^{*}$ is the set of all $f \in B^{*}$ such that $f(x) \geq 0$ for all $x \in B^{+}$. An element $f$ of $\left(B^{*}\right)^{+}$ is said to be order-continuous if $x=\sup \left(x_{i}\right)$ for an increasing net of positive elements $\left(x_{i}\right)$ implies $f(x)=\sup f\left(x_{i}\right)$. The set of all

order-continuous elements of $\left(B^{*}\right)^{+}$is denoted by $B^{\circ C}$. As in the case of $C[0,1]$, it is possible that $B^{O C}$ can contain only the zero functional. On the other hand, if the norm of $B$ is order-continuous, then $B^{O C}=\left(B^{*}\right)^{+}$.

An element $a$ of $B^{+}$is said to be $(\underline{O C})$-quasi-interior if $f(a)=0$ and $f \in B^{O C}$ imply $f=0$.

Now we set

$$
B(G)=\{x \in B: \phi(x)=x \text { for all } \phi \in G(B)\}
$$

and

$$
B(S)=\{x \in B: \phi(x)=x \text { for all } \phi \in S(B)\} \text {. }
$$

If $B(S)$ contains an (oc)-quasi-interior point, $B$ is said to be $H$-finite; otherwise, $B$ is called $H$-infinite.

\section{Problems.}

When $B$ is a Banach lattice, we have

$$
Z(B)=\{T \in L(B):|T x| \leq \lambda|x| \text { for all } x \in B \text { and some } \lambda\} \text {. }
$$

It is known ([2], Theorem 3.2) that, when $B$ is o-complete, $T \in Z(B)$ if and only if $T$ commutes with all band projections.

(2.1) When $B$ is a o-complete Banach lattice, we have $S(B)=\{1\}$, where 1 denotes the identity operator.

Proof. Let $\phi \in S(B)$. Since $Z(B)$ contains all band projections, $\phi$ commutes with all band projections. Hence, $\phi \in Z(B)$. Now, $B$ is 
orthogonally decomposable, has the Robinson property and norm, together with the dual norm, is absolutely monotone. Therefore, $\|\phi||=|| \phi \mid\|_{0}=1$ and, hence, $0 \leq \phi(x) \leq x$ for all $x \in B^{+}$. Similarly, $0 \leq \phi^{-1}(x) \leq x$ for all $x \in B^{+}$. Hence, $\phi=1$.

Now, let $B$ be a general ordered Banach space. The above fact leads to the following question : is $B$ lattice-ordered if $S(B)=\{1\}$ ? More generally, we shall consider the following problem.

Problem 1. Is $B(S)$ lattice-ordered?

We shall show that the answer is affirmative for a special class of ordered Banach spaces. This shows that, as $B$ becomes more latticelike, $S(B)$ will become smaller and $B(S)$ will become larger. An ordered Banach space is called an antilattice if $z=\sup (x, y)$ implies $x \geq y$ or $x \leq y$. (See [11] and [13].) Then, $B(S)$ will be the smallest when $B$ is an antilattice. We consider this problem in the following three forms.

Problem 2. If $B$ is an H-finite antilattice, is $B(S)$ generated by a single (oc)-quasi-interior point?

Problem 3. If $B$ is an $H$-infinite antilattice, do we have $B(S)=\{0\}$ ?

Problem 4. If $S(B)=G(B)$, is $B$ an antilattice?

3. Ordered Banach spaces of type (P).

Let $B$ be an ordered Banach space. We suppose that there is a family $\left\{P_{a}: a \in B^{+}\right\}$of projections (the idempotent elements of $L(B)$ ). An orthogonal decomposition $a=b-c$ is called proper if the following two conditions are satisfied:

$$
P_{b}(c)=P_{c}(b)=0 \text {; }
$$

(2) If $\phi(a)=a$ for some $\phi \in S(B)$, then $\phi(b)=b$.

For every $a \in B^{+}$, we set

$$
B_{a}^{+}=\left\{x \in B^{+}: f(x)=0 \text { if } f \in B^{O C} \text { and } f(a)=0\right\} \text {. }
$$


An ordered Banach space $B$ is said to be of type (P) if it is regular and it is equipped with a family $\left\{P_{a}: a \in B^{+}\right\}$such that the following conditions are satisfied:

(P1) $P_{a}\left(B^{+}\right)=B_{a}^{+}$and $\quad a \in B_{a}^{+}$for all $\quad a \in B^{+}$

(P2) If $a \in B(S)$, then $P_{a} \leq 1$.

(P3) Every element of $B$ admits a proper decomposition.

Before proceding further, we give some examples.

Example 1. Banach lattices which are $\sigma$-complete and in which the norms are order-continuous are of type (P). In this case, $P_{a}$ is defined by

$$
P_{a}(x)=\sup _{n \geq 1}(x \wedge n a)
$$

which is the band projection associated with $\{a\}^{\perp \perp}$. By definition, the norms of Banach lattices are absolutely monotone. Hence $B$ is regular. Since the norm is order-continuous, $B^{O C}=\left(B^{*}\right)^{+}$, and $B_{a}^{+}$coincides with the "positive bipolar" considered in [14] where the equality (P1) has been proved. (P2) and (P3) follow immediately from (2.1) and the basic properties of band projections.

Example 2. Let $M$ be a von Neumann algebra and $B=M^{h}$ be the ordered Banach space of all hermitian elements of $M$. Then $B$ is of type (P). First note that, this is obviously regular. For each $a \in B^{+}$, define $P_{a}$ by $P_{a}(x)=s(a) x s(a)$, where $s(a)$ is the support of $a$. Since $B^{O C}=\left(M_{*}\right)^{+}$, the positive part of the predual $M_{*}, f(a)=0$ for $f \in B^{O C}$ implies $f(s(a))=0$, and the relation (P1) can be proved directly or by a modification of a result in [5], p. 357. As to (P2), we first note that $T \in Z(B)$ if and only if $T 1 \cap M \in M^{\prime}$ and $T x=x \cdot T 1$ for every $x \in B$. For the proof of this fact, see, for instance, [1]. Since $M^{h}$ is orthogonally decomposable and has the Robinson property, $G(B)$ is the set of all bipositive isometries on $M^{h}$. In other words, $G(B)$ is the set of restrictions, to $M^{h}$, of all Jordan *-isomorphisms 
$\phi$ of $M$ such that $\phi(1)=1$. Furthermore, it follows from the above characterization of $Z(B)$ that $\phi \in S(B)$ if and only if $\phi$ is the restriction of a Jordan $\star-i s o m o r p h i s m$ of $M$ which is identical on the centre $M \cap M^{\prime}$. Hence, we have $B(S)=\left(M \cap M^{\prime}\right)^{h}$. Therefore, if $a \in B(S)$, then $s(a)$ is an central projection. Hence, $P_{a}(x) \leq x$ for all $x \in B^{+}$. Finally, the condition (P3) is satisfied because the usual orthogonal decomposition $a=a^{+}-a^{-}, a^{+} a^{-}=0$, is a proper decomposition.

Example 3. Let $M$ be a von Neumann algebra on a Hilbert space $H$ and suppose that there is a cyclic and separating vector $\xi_{0} \in H$ for $M$. Then, by the Tomita-Takesaki theory, there are a conjugation operator $J$ and a modular operator $\Delta$ associated with $\xi_{0}$. The real part of $H$,

$$
H^{J}=\{\xi \in H: J \xi=\xi\},
$$

is then an ordered Hilbert space ordered by the "natural cone"

$$
\left.H^{+}=\overline{\left\{\Delta \bar{\Delta}^{\frac{T}{4}} x \xi_{0}: x \in M^{+}\right.}\right\} \text {. }
$$

(See [6] and [9].) This is of type (P). Since the norm is absolutely monotone, $H^{J}$ is regular. We define $P_{\xi}$ by $P_{\xi}=p_{\xi}\left(p_{\xi}\right)$, where $p_{\xi}$ is the projection on the subspace $\left[M^{\prime} \xi\right]$ and $j\left(p_{\xi}\right)=J p_{\xi} J$. By [9], Theorems 4.5 and 4.6 , we have

$$
P_{\xi}\left(H^{+}\right)=\left\{n \in H^{+}:(n, \rho)=0 \text { if } \rho \in H^{+} \text {and }(\rho, \xi)=0\right\} \text {. }
$$

Therefore, we have the equality (P1) if the norm is order-continuous. To prove this, suppose that $\eta=\sup \left(n_{i}\right)$ for an increasing net $\left(n_{i}\right) \subset H^{+}$. Then, $-\eta \leq \eta_{i} \leq n$. The element $n$ can be assumed to be cyclic and separating, because otherwise we can take $n+\left(1-p_{\eta}\right) \dot{j}\left(1-p_{\eta}\right) \xi_{0}$ instead of $n$. Since $n \in H^{+}, H^{+}$is equal to the closure of $\left\{\Delta_{\eta}^{\frac{1}{4}} x \eta: x \in M^{+}\right\}$and there is an order isomorphism $\Phi$ of $M^{h}$ onto the set

$$
\left\{\rho \in H^{J}:-\lambda \eta \leq \rho \leq \lambda \eta \text { for some } \lambda\right\}
$$


defined by $\Phi(x)=\Delta_{\eta}^{\frac{3}{4}} x \eta \cdot\left([9]\right.$, Theorem 2.7.) since $\left(n_{i}\right)$ is contained in this set, there are $x_{i} \in M^{h}$ and $x \in M^{h}$ such that $\Phi\left(x_{i}\right)=n_{i}, \Phi(x)=n$ and $x=\sup \left(x_{i}\right)$. Furthermore, ||$x_{i} \xi-x \xi|| \rightarrow 0$ for every $\xi \in H$. Then, since ||$J\left(x_{i}-x_{\xi}\right) \| \rightarrow 0$,

$$
\begin{aligned}
\left\|n_{i}-n\right\|^{2} & =\left(\Delta_{n}^{\frac{1}{4}}\left(x_{i}-x\right)_{n}, \Delta_{n}^{\frac{1}{4}}\left(x_{i}-x\right)_{n}\right) \\
& =\left(\Delta_{n}^{\frac{3}{2}}\left(x_{i}-x\right)_{n},\left(x_{i}-x\right)_{n}\right) \\
& =\left(J\left(x_{i}-x\right)_{n},\left(x_{i}-x\right)_{n}\right) \\
& \leq\left\|J\left(x_{i}-x\right)_{n}\right\| \cdot\left\|\left(x_{i}-x\right)_{n}\right\| \rightarrow 0 .
\end{aligned}
$$

To prove (P2), we start with a result in [7] that $Z\left(H^{J}\right)=\left(M \cap M^{\prime}\right)^{h}$.

on the other hand, $G\left(H^{J}\right)$ is obviously the set of all unitary operators $u \in L(H)$ such that $u\left(H^{+}\right)=H^{+}$. Hence, $S\left(H^{+}\right)$is the set of all unitary operators $u$ in $R\left(M, M^{\prime}\right)$, the von Neumann algebra generated by $M$ and $M^{\prime}$, such that $u\left(H^{+}\right)=H^{+}$. Now, suppose that $\xi \in H^{J}(S)$. Then, $u j(u) \xi=\xi$ for all unitary element $u$ of $M$, because $u j(u) \in S\left(H^{J}\right)$. since $j(u) \xi=u^{*} \xi$, we have $\left[M^{\prime} \xi\right]=[M \xi]$, which means that $p_{\xi}$ is a central projection. Therefore, $p_{\xi} \leq 1$, and, hence, $P_{\xi} \leq 1$. The condition (P3) is satisfied because the usual orthogonal decomposition $\xi=\xi^{+}-\xi^{-},\left(\xi^{+}, \xi^{-}\right)=0$, is a proper decomposition.

\section{Problems 1,2 and 3 .}

We start with a lemma.

(4.1) Suppose that $B$ is an ordered Banach space which is regular and $B^{+}$is generating. Then, if $B$ is an antilatice and $0 \leq P=P^{2} \leq 1$, then $P=0$ or $P=1$.

Proof. Since $P \in 2(B)$ and $1-P \in 2(B)$, for each $a \in B^{+}$we have that $P a$ and $(1-P) a$ belong to a lattice-ordered subset of $B$, because $B$ is regular. Then, since $B$ is an antilattice, $P a$ and 
$(1-P) a$ must be comparable, that is, $P a \leq(1-P) a$ or $P a \geq(1-P) a$. It then follows that, for every $a \in B^{+}$, we have either $P a=0$ or $P a=a$. Suppose that there are nonzero elements $a$ and $b$ of $B^{+}$such that $P a=0$ and $P b=b$. Then, $P(a+b)=b$ and $a+b \neq b$, which is a contradiction. Hence, since $B^{+}$is generating, we have either $P=0$ or $P=1$.

We now give the answers to the first three problems when $B$ is of type (P).

(4.2) Let $B$ be an ordered Banach space of type (P).

(1). $B(S)$ is Zattice-ordered.

(2). If $B$ is an H-finite antilattice, $B(S)$ is generated by an (oc)-quasi-interior point.

(3). If $B$ is an $H$-infinite antilattice, $B(S)=\{0\}$.

Proof. (1). Let $a \in B(S)$ and $a=b-c$ be a proper decomposition. By (P3), $b \in B(S)$. Therefore, $P_{b} \leq 1$ by (P2). Furthermore, (P1) implies $P_{b} \geq 0$. Therefore, if $x \geq a$ and $x \geq 0$, we have $b=P_{b} a \leq P_{b} x \leq x$. This means $b=\sup (a, 0)$.

(2). Since $B(S)$ is a sublattice of an antilattice, it is totally ordered. Hence it is generated by a single element. since $B$ is $H$-finite, the element must be an (oc)-quasi-interior point.

(3). Suppose that $a \in B(S)$ and $a \neq 0$. By (P3), we can assume that $a \in B^{+}$. By (P1) and (P2), we have $0 \leq p_{a} \leq 1$. Hence by (4.1), we have $P_{a}=0$ or $P_{a}=1$. However, by (P1), $p_{a}=0$ implies $a=0$, a contradiction. Hence, $P_{\alpha}=1$, or, equivalently, $B_{a}^{+}=B^{+}$by (P1). Hence, $a$ is an (oc)-quasi-interior point. This contradicts the assumption.

An immediate consequence of (4.2) (1) is the following fact.

(4.3) When $B$ is an ordered Banach space of type $(P), S(B)=\{1\}$ implies that $B$ is Zattice-ordered. 


\section{Problem 4.}

The answer to this problem is in the negative. We shall give a negative example when $B$ is a finite-dimensional von Neumann algebra. We recall that every finite-dimensional von Neumann algebra $M$ is a direct sum

$$
M=M_{k_{1}} \oplus M_{k_{2}} \oplus \ldots \oplus M_{k_{m}},
$$

where $M_{k_{n}}$ is the algebra of all $k_{n} \times k_{n}$ matrices. The set $\left\{k_{1}, k_{2}, \ldots k_{m}\right\}$ characterizes the structure of $M$. We shall show that $G\left(M^{h}\right)=S\left(M^{h}\right)$ if and only if the numbers in this set are different.

(5.1) Let $M$ be a finite-dimensional von Nevmann algebra. Then, $G\left(M^{h}\right)=S\left(M^{h}\right)$ if and only if $M$ is a direct sum of factors which are not mutually Jordan *-isomorphic.

Proof. There are factors $M_{n}(n=1,2, \ldots m)$ such that $M$ is a direct sum : $M=M_{1} \oplus M_{2} \oplus \ldots \oplus M_{m}$. Since the centre of $M$ is the direct sum of centres of $M_{n}$, the central projections of $M$ are linear combinations of the following projections:

$$
\begin{gathered}
e_{1}=1 \oplus 0 \oplus \ldots \oplus, e_{2}=0 \oplus 1 \oplus \ldots \oplus 0, \\
e_{m}=0 \oplus \ldots \oplus 0 \oplus 1 .
\end{gathered}
$$

Now, suppose, for instance, that $M_{1}$ and $M_{2}$ are Jordan *-isomorphic and $\psi$ is the isomorphism. Then, the map defined by

$$
\phi\left(x_{1} \oplus x_{2} \oplus \ldots \oplus x_{m}\right)=\left(\psi^{-1}\left(x_{2}\right) \oplus \psi\left(x_{1}\right) \oplus x_{3} \oplus \ldots \oplus x_{m}\right)
$$

is a Jordan *-isomorphism of $M$ and $\phi\left(e_{1}\right)=e_{2}$. Therefore, $G\left(M^{h}\right) \neq S\left(M^{h}\right)$. To prove the converse, let $\phi \in G\left(M^{h}\right)$. Since $\phi$ preserves the minimal projections, $\phi$ maps the set $\left\{e_{1}, e_{2}, \ldots, e_{m}\right\}$ into itself.

If $\phi$ is not identical on the centre of $M$, we may suppose that $\phi\left(e_{1}\right)=e_{2}$. Then, a map $\psi: M_{1} \rightarrow M_{2}$ is defined by the following relation: 


$$
\begin{aligned}
\phi(x \oplus 0 \oplus \ldots \oplus 0) & =\phi\left((x \oplus 0 \oplus \ldots \oplus 0) e_{1}\right) \\
& =\phi(x \oplus 0 \oplus \ldots \oplus 0) e_{2} \\
& =0 \oplus \psi(x) \oplus 0 \oplus \ldots \oplus 0 .
\end{aligned}
$$

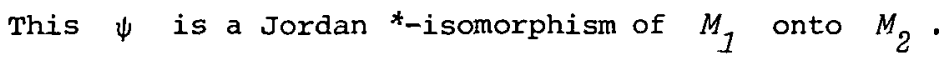

Remark. $G\left(M^{h}\right)=S\left(M^{h}\right)$ if and only if every bijective $0 . d$. homomorphism on $H$ is a normal operator. This, and the related problems on 0 . $d$. homomorphisms, will be discussed in a subsequent paper.

\section{References}

[1] E. Alfsen, Compact Convex Sets and Boundary Integrals, (SpringerVerlag, Berlin-Heidelberg-New York, 1971.)

[2] w. Arendt, "Spectral properties of Lamperti operators", Indicna University Math. J. 32 (1983), 199-215.

[3] W. Arendt, P. Chernoff and T. Kato, "A generalization of dissipativity and positive semigroups", $J$. Operator Theory 8 (1982), 167-180.

[4] C.J.K. Batty and D.W. Robinson, "Positive one-parameter semigroups on ordered Banach spaces", Acta Math. Applicondae 2 (1984), 221-296.

[5] O. Bratteli, T. Digernes and D.W. Robinson, "Positive semigroups on ordered Banach spaces", J. Operator Theory 9 (1983), 371-400.

[6] O. Bratteli and D.W. Robinson, Operator Algebras and Quantrom Statistical Mechonics I, (Springer-Verlag, Berlin-HeidelbergNew York, 1979).

[7] W. Bos, "A classification for selfdual cones in Hilbert space", Arch. Math. 30 (1978), 75-82.

[8] Cho-ho Chu and J.D.M. Wright, "Une theorie des types pour une classe d'espaces de Banach ordonnés", C. R. Acad. Sc. Paris, Serie A 281 (1975), 633-636.

[9] A. Connes, "Charactérisation des espaces vectoriels ordonnes sousjacents aux algèbres de von Neumann", Ann. Inst. Fourier, Grenoble 24 (1974), 121-155.

[10] R.V. Kadison, A representation theory for commutative topological algebras, (Memoirs of Amer. Math. Soc., No. 7, 1951). 
[11] R. V. Kadison, "Order properties of bounded selfadjoint operators", Proc. Amer. Math. Soc. 2 (1951), 505-510.

[12] R. V. Kadison, "Isometries of operator algebras", Ann. Math. 54 (1951), 325-338.

[13] W. A. J. Luxemburg and A. C. Zaanen, Riesz Spaces I, (North Holland, Aminterdam, 1971).

[14] D. W. Robinson and S. Yamamuro, "Hereditary cones, order ideals and half-norms", Pacific J. Math. 110 (1984), 335-343.

[15] W. Wils, "The ideal centre of partially ordered vector spaces", Acta Math. 127 (1971), 41-77.

[16] S. Yamamuro, "On linear operators on ordered Banach spaces", BuzZ. Austral. Math. Soc. 27 (1983), 285-305.

[17] S. Yamamuro, "On oxthogonally decomposable ordered Banach spaces", BuzZ. Aust. Math. Soc. 30 (1984), 357-380.

[18] S. Yamamuro, "Absolute values in orthogonally decomposable spaces", Bul2. Austral. Math. Soc. 31 (1985), 215-233.

Department of Mathematics

Institute of Advanced Studies

Australian National University

Canberra, A.C.T.

Australia.

Added in proof. 14 January, 1986. The fact, on p.179, that $T \in Z(B)$, for a Banach lattice $B$, if and only if $T$ commutes with all band projections was given first by W.A.J. Luxemburg in his lecture at the University of Arkansas in 1979. 